\section{REVELACIONES: LOS TEXTOS FOTOGRÁFICOS DE ELENA PONIATOWSKA}

\author{
AURORA CAMACHO DE SCHMIDT \\ Swarthmore College
}

\section{Aurora Camacho de} Schmidt

Amiga y estudiosa de la obra de Elena Poniatowska ha sido invitada a participar en el IV Congreso Trasatlántico realizado en la Universidad de Brown (2007). En diversos estudios afirma la necesidad de indagar en la poesía como medio para conocer a fondo el lenguaje. Entre sus ensayos destaca el dedicado a Nothing, nobody. The Voices of the Mexico City Earthquake (1999), así como otras investigaciones en torno a la voces indígenas en la poética de Octavio Paz y autores contemporáneos de México.
Porque la fotografía surge de la nada, de lo negro, del negativo y uno se queda temeroso al borde del precipicio porque bien puede ser una radiografía del alma.

(Elena Poniatowska, México sin retoque, 1987)

Desde la prosa profundamente lírica que comenta las fotografías de Richard Payne en Guerrero Viejo, pasando por las alegres y traviesas pláticas en torno a Juchitán de las mujeres y llegando a ese texto perturbador que se va entretejiendo con fotografías igualmente perturbadoras en El Niño, los niños de la calle, la literatura fotográfica de Elena Poniatowska ha llegado a constituir un atestiguamiento del México del siglo XX. Tanto en su literatura fotográfica como en sus novelas, ensayos y artículos periodísticos, el lenguaje de Poniatowska se caracteriza por su aparente sencillez, el resultado de una madura artesanía y la imaginación que recrea en vilo -no sólo el oído que registra- los matices más sutiles del lenguaje callejero, el de los camiones, los bares, las amigas que se llaman por teléfono, los campesinos que se aferran a la tierra o deciden irse al norte, los indígenas que celebran la vida a pesar de todo.

Este trabajo breve sostiene que los ensayos fotográficos de Elena Poniatowska nos revelan a México, pero lo hacen en forma extraordinariamente compleja. En los años en que se han publicado las presentaciones, prólogos, epílogos y comentarios a las colecciones de fotografías artísticas que hace la escritora, el campo de los estudios visuales ha crecido muy rápido, proponiendo nuevas exploraciones semióticas de la difícil tensión entre palabra e imagen. Bien conocido es el trabajo pionero de Walter Benjamin, Roland Barthes, Susan Sontag y John Berger, los pioneros que meditaron seriamente sobre lo que nos enseña una fotografía o una imagen artística. Más recientes son tentativas como la de Robert Coles, que a partir de la psiquiatría entra a definir la tarea documental como colaboración deliberada entre imagen fotográfica y lenguaje, colocando esa tarea en un terreno moral y político; de Frances Guerin y Roger Hallas, quienes trabajan sobre la relación entre trauma social y fotografía documental; de Mieke Bal, que con sus colaboradores ha inaugurado el campo de los «estudios visuales» a los que aplica algunos conceptos originales de su narratología literaria. Los estudios culturales han contribuido al campo de estudio de la fotografía y sus relaciones con el texto lingüístico, y esa bibliografía crece aprisa. Al mismo tiempo la crítica académica -especialmente la feminista- sobre el trabajo de Elena Poniatowska ha seguido aumentando, trayendo formas frescas de leer a la cronista, la actora política, la periodista que no para, la escritora de prólogos al por mayor, la novelista que escarba archivos y narra con deleite y asombro ${ }^{1}$.

Poniatowska misma ha hablado recientemente sobre su experiencia como escritora que colabora con fotógrafos, pero especialmente fotógrafas. Tanto con Mariana Yampolsky como con Graciela Iturbide, los pro-
1 La bibliografía contiene referencias a todos los autores arriba citados. de Elena Poniatowska

AURORA CAMACHO DE SCHMIDT 


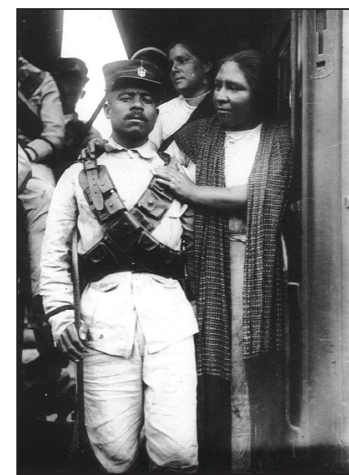

Soldaderas.

2

Dan Russek, «Literature and Photography, Parallel Crafts-An Interview with Elena Poniatowska» en Photography and Writing in Latin America: Double Exposures. Marcy E. Schwarz and Mary Beth Tierney-Tello, (eds.), Albuquerque, University of $\mathrm{New}$ Mexico Pres, 2006, p. 247.

3

John Berger, About Looking, New York, Pantheon, 1980, p. 58.

4

Alejandro Castellanos, "Fotografía contemporánea en Mexico», Presentación inaugural de la Exposición «Ríos de Luz», en la Wittliff Gallery de la South West Texas State University, San Marcos, Texas. Junio 23, 2002, p. 6.

5

Tita Valencia, «Elena Poniatowska y el tercer ojo: re-visión del México Profundo a través de sus prólogos a cuatro fotógrafas: Yampolsky, lturbide, Yovanovich, Sacabo y un zoom-in a Tina Modotti», manuscrito no publicado, 2005, p. 4

6

Beth Jörgensen, «Light Writing: Biography and Photography in Tinísima», Kristine Ibsen (ed.). The Other Mirror: Women's Narrative in Mexico, 1980-1995, Westport, CT/London: Greenwood Press, 1997, pp. 68-69.

Revelaciones: los textos fotográfico de Elena Poniatowska AURORA CAMACHO DE SCHMIDT yectos han sido concebidos como un todo. Ambas mujeres viajan juntas y se relacionan con los sujetos de las fotografías. Después, ante la selección de fotos, Poniatowska escribe. Dice ella:

What I have to do is come to my own conclusions, rather than describe what they have already said with the photos. It's all about parallel crafts, as if we were the two rails of a train: the photographer is one rail and the writer is the other, although I consider the role of the writer as substantially less important than that of the photographer in a book with text and images².

El interés de Elena Poniatowska en la fotografía se complementa con un profundo interés por la pintura. $\mathrm{Ha}$ escrito un libro sobre Juan Soriano y su novela epistolar Querido Diego, te abraza Quiela (1976) trata de Angelina Beloff, pintora rusa y compañera de Diego Rivera en París. Su texto sobre Frida Kahlo (1992) es un comentario a una colección fotográfica, pero también es la discusión de una gran pintora, la más famosa y original que haya tenido México y la esposa de Diego Rivera. Igualmente cuenta entre sus más conocidas entrevistas la que hizo en Lecumberri a David Alfaro Siqueiros, «el grande número tres» (Siqueiros, 18). El interés de la escritora por la fotografía extiende su gran interés por la pintura al mirar ambas como arte y como objetos culturales intrínsicamente relacionados con una historia y una geografía específicas.

Dos obras documentales, La noche de Tlatelolco (1971) y Nada, nadie: las voces del temblor (1988), contienen un rico material fotográfico puesto al servicio del texto. Aquí vemos a la periodista activamente reuniendo los dos medios para hacer su reportaje, sólo que el resultado es mucho más, tiene funciones que reconoce el crítico de artes visuales John Berger:

The task of an alternative use of photography is to incorporate photography into social and political memory, instead of using it as a substitute which encourages the athrophy of any such memory ${ }^{3}$.

Es claro que ambas obras han sido incorporadas a «una memoria social y política», pero no se trata de memorias lisas, consoladoras, cerradas. Son textos que inquietan y reclaman un trabajo por parte de los lectores. Alejandro Castellanos, director de la Casa de la Imagen, nos advierte al comentar la fotografía contemporánea de México:
Pensar utópicamente en que las fotografías funcionan como trincheras culturales para salvaguardar las identidades y las tradiciones locales es una quimera. Abrirse a la comprensión de un proceso múltiple, desigual y fragmentado es al parecer la única opción frente a la supuesta existencia de un imaginario mexicano coherente $e^{4}$.

Esta severa advertencia no se aplica a las dos obras mencionadas, que en sí mismas son colecciones de fragmentos desiguales, las voces de una comunidad múltiple. Pero pudieran impulsarnos a demandar una lectura crítica de la fotografía de los Alvarez Bravo, Iturbide, Yampolsky y Modotti, cuyas obras rescatan una nación que se pudiera llamar «auténtica» y aún inocente, o parte del «imaginario mexicano coherente». Sin embargo la dignidad personal que les infunde Mariana Yampolsky a los sujetos de sus fotografías ya subvierte la expectativa del mercado cultural. Como escribe Tita Valencia ${ }^{5}$, varias fotógrafas cuya obra ha sido prologada o comentada por Poniatowska tienen en común con ella el haber crecido fuera del país, «sin lastre», capaces de amar a México «con ojos niños de fuereña precoz». Es decir, ajenos al sistema de prejuicios sociales que les habría impedido a los mexicanos de cierta clase social encontrar tanta belleza y fuerza personal, tanto poder subjetivo, entre campesinos, indígenas, o niños de la calle.

La novela de Poniatowska Tinísima de 1992, testimonio biográfico sobre la fotógrafa italo-estadounidense Tina Modotti, expresa en múltiples formas el aprecio por la fotografía de la autora y su protagonista. Como ha visto Beth Jörgensen, Tina aparece en la novela como una mujer fuertemente dependiente de los hombres de su vida. Uno de ellos es Edward Weston, el fotógrafo de California que la lleva a México como ayudante y la convierte en su amante, mientras le enseña el arte de la fotografía. Jörgensen ${ }^{6}$ contrasta la pasividad de las fotografías que Weston toma de Tina desnuda con la intención social de sus propias fotos, como la de las manos de los trabajadores. En esta novela, texto fotográfico, el arte de Tina Modotti está íntimamente ligado a su amor por México, un amor que ella experimenta como búsqueda, y que eventualmente la lleva a su re-politización e independencia personal.

Dos ensayos, Las soldaderas (1999), con fotografías de la Revolución del archivo Casasola y Juchitán de las mujeres (1989), con las fotografías de Graciela Iturbide, ostentan 
una cualidad especial: las mujeres de clase baja de todo el país que se desplazaron con los combatientes y las zapotecas de Juchitán se ven como seres actuantes y eróticos, y su sexualidad se convierte en un centro de gravedad de la escritura. Ésta es una restauración a contrapelo de la conciencia dominante que tiende a deserotizar a la clase baja (a menos de que se trate de prostitutas) y a los grupos raciales minoritarios. De hecho ambos textos reinterpretan las historias oficiales.

El artículo que acompaña a Juchitán de las mujeres se titula «El hombre del pito dulce» y nos avisa de que: «los hombres tienen el pito dulce o salado según se apetezca y las mujeres están muy orgullosas de serlo, porque llevan su redención entre las piernas y le entregan a cada cual su propia muerte. 'La muerte chiquita' se llama el acto amoroso»’. La crítica escocesa Irene Matthews reconoce el esencialismo de las posiciones feministas de Poniatowska en Juchitán, pero agrega:

As photographed by Graciela Iturbide and expounded by Elena Poniatowska, the women of Juchitán exemplify certain deep assumptions about femaleness but also challenge the prevailing clichés (visual and rhetorical) that mark Mexico as a monolithic society built upon macho men and submissive, monotonously pregnant women 8 .

Hay un deseo de jugar, de encontrar la hilaridad de un pueblo oaxaqueño en donde las convenciones dejan de existir y la sexualidad parece vivirse sin preguntas ni problemas. No duda Tita Valencia en llamarla «la lujuria de Juchitán», quizá reflejada más en el lenguaje que en las fotografías. Tal falta de modestia nos recuerda las palabras de Susan Sontag en su introducción a la colección fotográfica de Annie Leibovitz titulada Women:

We want photography to be unmythic, full of concrete information. We are more comfortable with photographs that are ironic, unidealizing. Decorum is now understood as concealment. We expect the photographer to be bold, even insolent. We hope that subjects will be candid, or naïvely revealing (2000).

Igualmente algunas soldaderas se ven en las fotografías contentas junto a sus hombres. Su valentía legendaria se afirma nuevamente, su importancia para la Revolución: «Sin las soldaderas, los hombres llevados de leva hubieran desertado»?. Es preciso recordar que estas mujeres también tienen un papel en los procesos de cambio y resistencia.
«Hubo casos de mujeres que no esperaron a que llegaran las tropas rebeldes a sacarlas de la monotonía de su vida: fueron a su encuentro» ${ }^{10}$.

La investigadora británica Andrea Noble ofrece una crítica negativa del intento feminista de Poniatowska en Las soldaderas, al sugerir que su discusión no propone una reinscripción

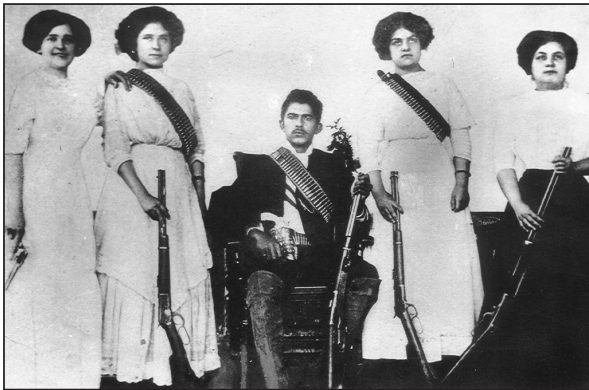

Soldaderas. compleja de la diferencia de género de estas mujeres en el grupo social de las tropas revolucionarias. En un artículo muy interesante cuyo tema principal es la fotografía de una mujer combatiente, sugiere que el ensayo poniatowskiano podría reducirse a un «ellas también estaban ahí» (Noble, 141). Sin embargo, el ensayo Las soldaderas muestra una particular complejidad. El texto, respaldado por una amplia investigación, organiza una recreación cultural del personaje de la soldadera y una reflexión original sobre sus múltiples papeles sociales, antes de abrirnos la puerta a la galería fotográfica. La misma autora ironiza que los corridos «previenen a las abnegadas soldaderas de sí mismas. No deben confiarse tanto ni ser tan buenas gentes. La vida es canija y también la vista engaña» ${ }^{11}$. La intención de Poniatowska no es afirmar la libertad de acción de estas mujeres, sino su múltiple presencia histórica y la falsedad de una historia que no las incluya.

Las soldaderas se preocupa por la memoria histórica. Entra en discusiones de lugar (geografía), tiempo (historia) y grupos sociales (identidad), creando una «construcción fotonarrativa» ${ }^{12}$ que va más allá de la corrección de una ausencia.

El breve ensayo fotográfico que sale de las categorías establecidas por todos los otros de Poniatowska se titula «La cárcel de los sueños de Vida Yovanovich» (1997) y comenta una colección estremecedora de fotografías del mismo título tomadas en un asilo de ancianos indigentes -posiblemente en la Ciudad de México- por la fotógrafa cubana de origen yugoeslavo ${ }^{13}$. La estrategia ensayística de la periodista consiste en centrarse en lo que Yovanovich ejecuta y logra a través de esta colección, en la cual ella misma es objeto de la cámara. Se afirma la valentía de la fotógrafa al mirar de frente su propia decrepitud futura, pero no encontramos una afirmación de la subjetividad de las mujeres ancianas en el texto. Hay algo que nos molesta en la exhibición de personas que probablemente no hayan
7

Graciela Iturbide y Elena Poniatowska, Juchitán de las mujeres, México, Ediciones Toledo, 1989, p. 11.

8

Irene Matthews, "Woman Wathching Women, Watching", Reinterpreting the Spanish American Essay: Women Writers of the 19th and 20th Centuries. Doris Meyer (ed.), Austin, University of Texas Press, 1995 p. 235

9 ras, México, ERA y CONACULTA, 1999, p. 14.

10

Ibid., p. 15.

11

Ibid., p. 29.

12

O «photonarrative construction». Este término aparece en el excelente ensayo introductorio de Alex Hughes y Andrea Noble a la colección de artículos titulada Phototextualities: Intersections of Photography and Narrative, Albuquerque, N.M., University of New Mexico Press, 2003, p. 4.

13

Le agradezco mucho a Tita Valencia la identificación y el envío de este libro.
Elena Poniatowska, Las soldade- 


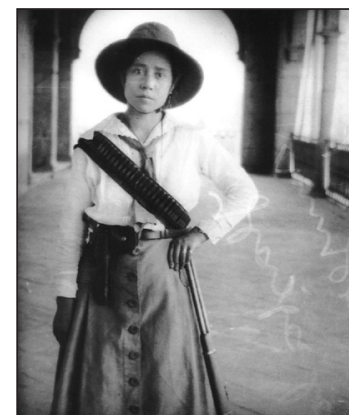

Soldadera.

14

Vida Yovanovich, Cárcel de los sueños, México, Fondo Nacional para la Cultura y las Artes, 1997.

15

Mark Reinhardt, Holly Edwards and Erina Duganne (eds.), Beautiful Suffering: Photography and the Traffic in Pain Williamstown, MA and Chicago, Williams College Museum of Art and University of Chicago Press, 2006, p. 7

16

Amy Conger and Elena Poniatowska, Compañeras de México: Women Photograh Women Riverside, University of California Riverside, 1990, p. 54

17

Mariana Yampolsky, La raíz y el camino, presentación de Elena Poniatowska, México, Fondo de Cultura Económica, 1985, p. 10.

18

Yampolsky, Imagen - Memoria I Image - Memory, textos de Elizabeth Ferrer y Elena Poniatowska, México, Consejo Nacional para la Cultura y las Artes, 1999, p. 173.

19

Amy Conger and Elena Poniatowska, op. cit., pp. 51-52.

20

Yampolsky, La raíz y el camino, p. 10.

Revelaciones: los textos fotográficos de Elena Poniatowska

AURORA CAMACHO DE SCHMIDT tenido la capacidad de consentir ser objeto de fotografías publicadas, como no son dueñas de su decisión de estar ahí, en un almacén de gente que espera pasivamente la muerte. De repente una afirmación como "Aquí también los ancianos se mueren de sí mismos, de necesidad, de desamor. Solos se matan y solos se van muriendo» nos recuerda quién está escribiendo ${ }^{14}$. Pero el proyecto entero pertenece al mundo de la exhibición fotográfica reciente (Williams College, 2006) que se tituló Beautiful Suffering: Traffic in Pain. La introducción al lujoso libro que resultó dice:

Without injured bodies and devastating landscapes, without scenes of death, destruction, misery, and trauma, the contemporary image environment would be virtually unrecognizable. These scenes of affliction are often formally striking or beautifully rendered: every day, without much effort, one may come across exquisite images of other people's suffering 15 .

En «La cárcel de los sueños» no hay visión crítica. Indudablemente se trata de un proyecto artístico legítimo, pero no de una relación social entre iguales. La mediación que a menudo ejerce la ensayista con su poderoso lenguaje le cede el foro a una mirada que convierte en objetos los cuerpos -a veces desnudos- de las ancianas para permitir una indagación personal al final de la colección, cuando la imagen fotográfica de la fotógrafa y sus espejos convierte el proyecto en un dilatado «memento mori».

La escritura fotográfica de Poniatowska contiene un elemento celebratorio de la participación numerosa de mujeres en el arte de la fotografía. Escribe en Compañeras (1990):

Sin embargo entre las fotógrafas se da una unión secreta, fisiológica, como entre las escritoras y resulta mucho más evidente que el lazo entre los hombres artistas en cualquier campo que se la pasan metiéndose zancadillas ${ }^{16}$.

Probablemente la colaboración más continua y entrañable de la escritora se haya dado con Mariana Yampolsky, la fotógrafa de La raíz y el camino (1985), Mazahua (1993), La casa en la tierra (1980), Mariana Yampolsky y la buganvillia (2001), el libro bilingüe Imagen-Memoria / Image-Memory (1999), y el libro en inglés The Edge of Time (1998), todos presentados sensitivamente por su amiga Elena. El trabajo de Yampolsky inspira respeto, invita al silencio. Está bañado en una sobriedad modernista y minimalista, $y$ en algunos casos refleja la fascinación por la forma natural pura de Weston. Por otro lado, la pobreza y el aislamiento de muchas láminas constituyen una mirada desaprobatoria. Nos recuerdan que Yampolsky es hija del Taller de la Gráfica Popular, sitio privilegiado del legado revolucionario de México. Hay un contenido político en estos álbumes.

Los ensayos de Poniatowska que se yuxtaponen a las imágenes están llenos de aciertos verbales que a menudo retratan a la escritora tanto como a la fotógrafa, como cuando hace decir a Yampolsky en un arrebato de entusiasmo: «Este es mi país! $»^{17}$, o cuando declara «Más que ningún otro fotógrafo es Mariana Yampolsky quien se ha acercado al impulso vital que singulariza a nuestro país, su poderoso afán de supervivencia, la colosal hazaña de llegar hasta la noche» ${ }^{18}$. O bien «[la de Yampolsky es] una de las miradas más inteligentes y honestas de nuestra cultura» ${ }^{19}$. $\mathrm{O}$ «va por caminos pobres y calurosos de un México libremente escogido» ${ }^{20}$. Todo esto se podría decir de ella misma.

La destreza de Elena Poniatowska como entrevistadora queda en evidencia en $\mathrm{Ma}$ riana Yampolsky y la buganvillia donde la periodista deja hablar a la fotógrafa, recortando, pegando, integrando como suele hacer, dándonos una autoexposición diegética de la artista. Los lectores reciben una historia compleja y ágil. Entre la creadora de las imágenes y la artesana de las palabras se produce una complicidad en que la última parece decir: «quiero que todos vean lo que tú ves, como tú lo ves. Y además...»

En El Niño, los niños de la calle (1999), producido en colaboración con el fotógrafo suizo Ken Klich, el texto impresiona por su ritmo lento, su tempo pausado, a diferencia de los torbellinos verbales de La noche de Tlatelolco o Nada, nadie. La autora transcribe preguntas hechas a un grupo de niños y adolescentes que ha tomado una casa abandonada cerca de la estación de Buenavista. Como menores de edad, es fácil verlos como víctimas de la violencia familiar y social; la sociedad los mira con recelo por su capacidad de volverse ellos mismos productores de desorden y violencia. Pero el texto no fue creado para satisfacer nuestra pregunta: ¿qué va a pasar con esos niños? ¿los van a llevar a un refugio u orfelinato? Nos enteramos, sí, de que todos inhalan solventes en trapos que pueden arder fácilmente, de que se roban uno a otro cualquier objeto que despierte su codicia, y que es común entre ellos la prostitución 
con extraños y el intercambio sexual dentro del grupo. Pero no hay juicios de valor sobre estas condiciones calamitosas. En vez de eso hay preguntas como ¿te gusta vivir aquí? ¿conoces a todos los chavos? Y este mero acto reorganiza las premisas de nuestra lectura.

Podríamos pensar que la larga práctica periodística le permite a Poniatowska acercarse a los niños de esta manera. Hay algo más: un respeto radical por los sujetos con quienes habla, actitud que en sí constituye un acto crítico.

Barthes llega a decir que el comentario textual de la fotografía la convierte en una institución social, un signo que expresa a su sociedad, habiéndose originado simplemente como un arte mecánica. Pero las ilustraciones con que trabaja Elena Poniatowska ya tienen un comentario implícito y están lejos de ser un doble objetivo de la realidad, especialmente tratándose de Yampolsky. Además, la escritora teje una red contextual que impulsa la interpretación del corpus fotográfico que discute. Sus estrategias ensayísticas les prestan a los textos gracia y peso a la vez. Puede empezar la discusión en la mitad de una conversación, incorporando a su discurso los dichos, refranes, giros o vocabulario del pueblo.

Todos saben, por ejemplo, que Poniatowska es la maestra de las enumeraciones, las cadenas potencialmente infinitas de elementos afines y a veces caóticos, como en estos párrafos:

Según Jesusa las mujeres de la Revolución fueron llamadas vivanderas, comideras, galletas de capitán, soldaderas, chimiscoleras, soldadas, juanas, cucarachas, argüenderas, mitoteras, busconas y hurgamanderas ${ }^{21}$.

\section{Y en Juchitán de las mujeres:}

Y más allá, palmas, escobas, sogas, medias sogas, y más aca, huaraches, estribos, fustes, sillas, espuelas, y aquisito chapas, bisagras, cerrojos que se parecen a los camarones redondos, cerrados sobre sí mismos, amontonados en canastas, traídos por los huaves, junto con los frescos huevos de tortuga y el pescado seco, tatemado al sol. Allasito los totopos istmeños, esas amplias tortillas...22.

Y todo esto no tiene fin; apenas estamos empezando el libro. Son series interminables que se paran arbitrariamente, llevando el texto a una oralidad agradable, casera, del pueblo.
En «Microfotografía» Elena Poniatowska arguye que Manuel Álvarez Bravo es capaz de retener fotografías exactas en el cerebro. No sólo eso, es capaz de revelarlas directamente de su cabeza. Así dice:

Manuel echa el revelador, nos debatimos en vano, el fotógrafo nos ha aprisionado. Manuel Alvarez Bravo tiene la llave de la trampa para dejamos salir, clic, pero no quiere, clic, encierra, clic, imprime, clic, nos fija, clic, nos expone en unos cuantos segundos y desentraña nuestra negra conciencia. . .23

Aún en los más serios ensayos, la escritura juega, se regocija, se hace presente e íntima para marchar de la mano con la presente presencia de la foto. En Mazahua, por ejemplo, Poniatowska no sólo responde a las láminas de Yampolsky, sino además habla con la gente que ella ha fotografiado o está por fotografiar. «Y ahora, ¿qué puede usted hacer con un solo brazo?, pregunto atolondrada. Y responde 'Lo que puedo hacer es sufrir'. En parte es cierto, pero don Inocencio con su único brazo es el único tejedor de San Felipe Santiago y si se sienta frente a su telar, puede terminar un gabán en una semana» ${ }^{24}$. Aquí podemos ver cómo el lenguaje surge de relaciones personales, comunicaciones entre dos sujetos. Antes de que llegue a la página ya ha sido lenguaje compartido.

Después de caminar por estos textos, vale la pena recordar que para Barthes no podemos procesar una fotografía sin recurrir al lenguaje $\mathrm{e}^{25}$. Las fotografías dicen, pero su decir es a veces contradictorio. Según Poniatowska la cámara habla. Comentando la obra de Héctor García, nos asegura:

A lo largo de 25 años de trabajo no recuerdo haberle oído un «Mirando para allá, por favor» 0 «Voltee usted para acá.» El fotógrafo no dice una palabra, pico de cera, es la cámara la que habla su duro lenguaje de vida vivida ${ }^{26}$.

Y en otra parte habla de los grandes muralistas de Mexico como los autores de "pintura testimonial... o la crónica de su tiempo».

Estas citas podrían llevarnos a creer que la labor de la escritora es una simple traducción $^{27}$. Sí se trata de un traslado entre dos medios, pero Poniatowska no «lee» obedientemente la fotografía. Más bien, comparte la mirada de su cómplice fotógrafo o fotógrafa, dialoga con ella, libera sus potencialidades, la hace hablar. Entre la sintaxis de la lengua española y la estructura de la imagen visual hay
21

Poniatowska, Las soldaderas, p. 22.

22

Iturbide y Poniatowska, Juchitán de las mujeres, p. 14.

23

Fomento Cultural Banamex, Ma nuel Álvarez Bravo: el artista. su obra y sus tiempos, textos de Elena Poniatowska, México, Fomento Cultural Banamex, 1991 , p. 27.

24

Yampolsky, Mazahua, texto de Elena Poniatowska, Toluca, Edo. De México, Gobiemo del Estado de Mexico, 1993, p. 15.

25

Roland Barthes, "The Photographic Message», en A Barthes Reader, edited and introduced by Susan Sontag, New York, Hill and Wang, 1982, pp. 194 y ss.

26

Héctor García, México sin retoque, presentación de Elena Poniatowska, México, Universidad Nacional Autónoma de México, Coordinación de Difusión Cultural, Dirección de Literatura, 1987, p. 4

27

Mieke Bal y Joanne Morra discuten el importantísimo papel de la traducción en su editorial a un volumen de la revista académica "Journal of Visual Culture». En muchas maneras ellas son las críticas teóricas cuyo trabajo mejor se aplica al acto "traduciente» de Elena Poniatowska. Dicen ellas: "We are using the term "in termedial translation» to mean, quite simply, translating across media. To 'translate across' is to work within discourses and practices of intertextuality, intersemiotics and interdisciplinarity, which can lead to movements across genres, media, bodies of knowledge and subjects. More figuratively, translating across is concerned with the marginal, the gaps, fissures and contradictions of working in the interstices between these various boundaries.» «Editorial: Acts of Translation. Journal of Visual Culture 6.1 (2007), 2-3.

\footnotetext{
Revelaciones: los textos fotográficos de Elena Poniatowska AURORA CAMACHO DE SCHMIDT
} 


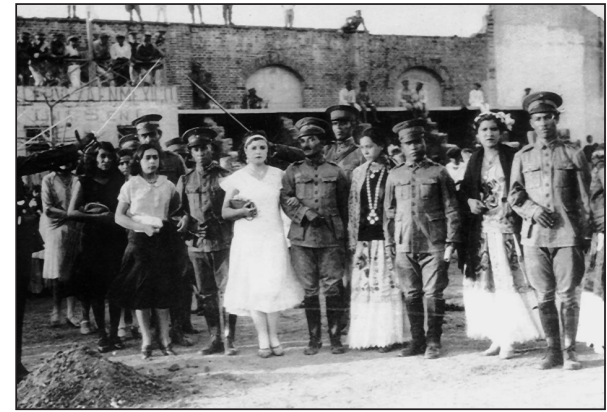

Soldaderas

28

Aníbal González, Journalism and the Development of Spainsh American Narrative, Cambrdge, Cambridge University Press, 1993, p. 125.

Revelaciones: los textos fotográficos de Elena Poniatowska AURORA CAMACHO DE SCHMIDT una grieta insalvable, no una bisagra. Solamente una escritora que se sepa dueña de la lengua española y honda conocedora de los proyectos de sus colegas fotógrafos es capaz de soltar la energía que guarda la imagen. Que éste es un poder periodístico lo dice mejor que nadie Aníbal González al afirmar que la búsqueda de Poniatowska es una búsqueda del origen, que inevitablemente empieza con el impulso periodístico de entender el presente ${ }^{28}$.

Elena Poniatowska puede hacerlo porque esta tentativa es parte de una trayectoria que consiste en atestiguar la vida de su patria dándole atención a los pobres, los obreros, los niños de la calle, los sirvientes, los campesinos, los presos, los indígenas, los emigrantes, y sobre todo a las mujeres de todos esos grupos en toda su especificidad corporal, y no como directiva moralista y abstracta. Esa atención está encarnada en un lenguaje lleno de color, textura, humor, travesura, oralidad, domesticidad; un lenguaje rico en tradiciones populares, especialmente femeninas. La palabra que rodea a la fotografía se vuelve luz, se hace revelatoria. Denuncia y canta al mismo tiempo.

En el momento actual de México, con más de cien millones de mexicanos, la mitad de los cuales viven en extrema pobreza o han tenido que emigrar a Estados Unidos, el trabajo revelatorio se vuelve urgente. Los textos fotográficos de Elena Poniatowska reafirman el poder interpretativo de una mujer mexicana. Son textos que nos reconectan -lectores nacionales e internacionales- a un lugar y a su gente. Esa gente encarna un sistema de signos ajenos cuyos códigos hemos olvidado, si algún día los supimos. Elena Poniatowska encuentra la llave y nos la presta. Con su gracia y su risa, su seriedad y su travesura, su insistencia y su luminosidad, los ensayos fotográficos de Elena Poniatowska nos dan en último análisis la medida de nuestra humanidad.

\section{BIBLIOGRAFÍA}

Amador Gómez Quintero, Raysa E. and Mireya Perez Bustillo. The Female Body. Perspectives of Latin American Artists. Westport, Connecticut-London: Greenwood Press, 2002.

Bal, Mieke. «Reading Art». A Mieke Bal Reader, Chicago: Chicago University Press, 2000.
Barthes, Roland. "The Photographic Message.» In $A$ Barthes Reader. Edited and introduced by Susan Sontag. New York: Hill and Wang, 1982.

Benjamin, Walter. Illuminations. Edited and with an Introduction by Hannah Arendt. Translated by Harry Zohn. New York: Schocken Books, 1983.

Berger, John. About Looking. New York: Pantheon, 1980.

Castellanos, Alejandro. «Fotografía contemporánea en Mexico». Presentación inaugural de la Exposición «Ríos de Luz», en la Wittliff Gallery de la South West Texas State University, San Marcos, Texas. Junio, 2002.

Coles, Robert. Doing Documentary Work. Oxford-New York: Oxford University Press, 1997.

Conger, Amy and Elena Poniatowska, Compañeras de México: Women Photograb Women, Riverside: University of California Riverside, 1990.

Consejo Nacional para la Cultura y las Artes, Siqueiros en Lecumberri: una lección de dignidad 1960-1964. Entrevista de Elena Poniatowska. Mexico: Consejo Nacional para la Cultura y las Artes e Instituto $\mathrm{Na}$ cional de Bellas Artes, 1999 .

Fomento Cultural Banamex, Manuel Álvarez Bravo: el artista. su obra y sus tiempos. Textos de Elena Poniatowska. México: Fomento Cultural Banamex, 1991.

Frida Kablo: the Camera Seduced. Memoir by Elena Poniatowska. Essay by Carla Stellweg. San Francisco: Chronicle Books, 1992.

García, Héctor. México sin retoque. Presentación de Elena Poniatowska. México: Universidad Nacional Autónoma de México, Coordinación de Difusión Cultural, Dirección de Literatura, 1987.

González, Aníbal. Journalism and the Development of Spainsh American Narrative, Cambridge: Cambridge University Press, 1993.

Guerin, Frances and Roger Hallas (eds.). The Image and the Witness: Trauma, Memory and Visual Culture. London / New York: Wallflower Press, 2007.

Hughes, Alex and Andrea Noble (eds.). Phototextualities: Intersections of Photography and Narrative. Albuquerque, N.M.: University of New Mexico Press, 2003.

Iturbide, Graciela y Elena Poniatowska. Juchitán de las mujeres. México: Ediciones Toledo, 1989.

Jörgensen, Beth E. The Writing of Elena Poniatowska: Engaging Dialogs, Austin: University of Texas Press, 1994. 
Jörgensen, Beth. «Light Writing: Biography and Photography in Tinisima.» Kristine Ibsen, ed. The Other Mirror: Women's Narrative in Mexico, 1980-1995. Westport, CT /London: Greenwood Press, 1997.

Klich, Kent and Elena Poniatowska. El Niño: Children of the Street. Niños de la calle. Ciudad de México. Series Image and Imagination. Syracuse: Syracuse University Press, 1999.

Matthews, Irene. «Woman Wathching Women, Watching.» Reinterpreting the Spanish American Essay: Women Writers of the $19^{\text {th }}$ and 20th Centuries. Doris Meyer, ed. Austin: University of Texas Press, 1995.

Payne, Richard and Elena Poniatowska. Guerrero Viejo. Translated by Deanna Heikinnen. Houston, Texas: Anchorage Press, 1997.

Pino Ojeda, Walescka. Sobre castas y puentes: conversaciones con Elena Poniatowska, Rosario Ferré y Damiela Eltit. Chile: Editorial Cuarto Propio, 2000.

Poniatowska, Elena. Mariana Yampolsky y la buganvillia. México, D.F. : Plaza y Janés, 2001.

- Mexican Color. Photographs by Amanda Holmes. Translated by Aurora Camacho de Schmidt. New York: Stewart, Tabori and Chang, 1998.

- Las soldaderas. México: ERA y CONACULTA, 1999.

- Todo empezó el domingo. Dibujos de Alberto Beltrán, México, Fondo de Cultura Económica, 1993.

- El último guajolote. (Memoria y olvido: Imágenes de México). México: Secretaría de Educación Publica, 1982.

Reinhardt, Mark, Holly Edwards and Erina Duganne, eds. Beautiful Suffering: Photography and the Traffic in Pain. Wil- liamstown, MA and Chicago: Williams College Museum of Art and University of Chicago Press, 2006.

Russek, Dan. «Literature and Photography, Parallel Crafts. An Interview with Elena Poniatowska.» Photography and Writing in Latin America: Double Exposures. Marcy E. Schwarz and Mary Beth Tierney-Tello, eds. Albuquerque: University of New Mexico Pres, 2006.

Shawcross, Nancy. «Mythologies of the Photograph.» In Roland Barthes on Photography: the Critical Tradition in Perspective. Gainesville: University of Florida Press, 1997.

Valencia, Tita. «Elena Poniatowska y el tercer ojo: re-visión del México Profundo a través de sus prólogos a cuatro fotógrafas: Yampolsky, Iturbide, Yovanovich, Sacabo y un zoom-in a Tina Modotti». Manuscrito no publicado. 2005.

Yampolsky, Mariana. The Edge of Time: Photographs of Mexico. Foreword by Elena Poniatowska. Introduction by Sandra Berler. Afterword by Francisco Reyes Palma. Austin, Texas: University of Texas Press, 1998.

- Imagen-Memoria / Image-Memory. Textos de Elizabeth Ferrer y Elena Poniatowska. México: Consejo Nacional para la Cultura y las Artes, 1999.

- Mazabua. Texto de Elena Poniatowska. Toluca, Edo. De México: Gobiemo del Estado de México, 1993.

- La raíz y el camino. Presentación de Elena Poniatowska. México: Fondo de Cultura Económica, 1985.

Yovanovich, Vida. Cárcel de los sueños. México: Fondo Nacional para la Cultura y las Artes, 1997. 